\title{
ANÁLISIS ESPACIAL DE LA FECUNDIDAD ADOLESCENTE EN MUNICIPIOS DE CHIAPAS
}

\author{
Spatial Analysis of Adolescent Fertility in Chiapas Municipalities
}

\author{
Gerardo Núñez-Medina \\ Hilda María Jiménez-Acevedo
}

Resumen: La finalidad de este trabajo es analizar la distribución espacial de la fecundidad de adolescentes entre 10 y 19 años y algunos de sus determinantes más importantes en Chiapas en 2015. La importancia de esta investigación radica en tres factores: 1) Chiapas es el estado con mayor proporción de población joven-adolescente en el país; 2) es la tercera entidad con el mayor número de embarazos adolescentes, y3) 85 de sus 118 municipios fueron catalogados por CONAPO como de alta y muy alta marginación, lo que hace evidente que la entidad presenta importantes problemas de segregación económica, social y espacial. En este trabajo se busca aportar información relevante sobre el comportamiento y determinantes de la fecundidad en este grupo de edad, tema poco estudiado.

Palabras clave: distribución geográfica, fecundidad, jóvenes, marginación, analfabetismo, índice de Moran.

Abstract: The purpose of this study is to analyze the behavior of the spatial distribution of adolescent fertility and some of its most important determinants in 2015 in Chiapas. The importance of this research lies in three fundamental factors: (1) Chiapas has the highest percentage of adolescents and teenagers in Mexico; (2) It has the third highest number of adolescent pregnancies; (3) CONAPO has classified 85 of its 118 municipalities as having high or very high marginalization. Thus, the state has important economic, social, and spatial-segregation problems. Further, this paper provides relevant information on how the fertility of this age group has evolved, a subject that has been scarcely studied.

Keywords: geographical distribution, marginalization, fertility, youth, Moran index.

Gerardo Núñez Medina. Doctor en Demografía por la Universidad de Barcelona, España. Profesor de asignatura en la Universidad Autónoma de Chiapas, México. Temas de especialización: Estudios de población. Correo electrónico: gerardo.nml@gmail.com.

Hilda María Jiménez Acevedo. Doctora en Desarrollo Social por la Universidad de Londres, Reino Unido. Profesora de tiempo completo en la Universidad Autónoma de Chiapas, México. Temas de especialización: pobreza y políticas públicas. Correo electrónico: luzhec10@hotmail.com.

Enviado a dictamen: 24 de enero de 2017. Aprobación: 30 de octubre de 2017. Revisiones: 2. 
E l incremento observado en los últimos años de los embarazos en adolescentes se ha convertido en una preocupación creciente para muchos gobiernos y diseñadores de políticas públicas debido principalmente a que este incremento se contradice con eventos como: el descenso histórico de la tasa global de fecundidad, el aumento de los niveles de educación en la población femenina, la mayor disposición de información sobre prevención de embarazos, el acceso casi universal a métodos anticonceptivos y la implementación de políticas públicas de planificación familiar. Este preocupante fenómeno resulta complejo de atender posiblemente por implicaciones asociadas a aspectos demográficos, de diseño y operación de los programas de gobierno sobre salud pública y derechos - reproductivos y humanos-, y otros aspectos relacionados con los ingresos y el nivel de bienestar.

Para comprender el fenómeno de la fecundidad en menores de 19 años es necesario conocer el contexto en que se produce y los factores asociados a él, como la educación, el género, la etnia, la condición económica, la cultura, el espacio geográfico de residencia y la religión, entre otros. ${ }^{1}$

En general, los determinantes del embarazo adolescente se relacionan con contextos de pobreza y vulnerabilidad asociados al género y a la edad, además de a aspectos socioculturales. Adicionalmente, los embarazos adolescentes producen importantes efectos en la vida de las madres, de sus familias y de su descendencia porque contribuyen a reproducir la pobreza y la falta de oportunidades. El hecho se vincula con aspectos educativos, de ingresos, de salud, ${ }^{2}$ de costumbres y de violencia y abusos, elementos que coartan la libertad y el desarrollo pleno de las adolescentes (Gómez y Hernández, 2002; Climent, 2003).

Carlos Welti (2016) afirma que, si bien el embarazo adolescente se relaciona con grupos en condiciones socioeconómicas desventajosas y con bajos niveles educativos, esta situación ya no es exclusiva de ellos, porque en áreas urbanas la maternidad aumenta entre mujeres jóvenes con educación media o media superior, lo que se explica por la falta de oportunidades de desarrollo en otros ámbitos: "estudiar o terminar una carrera no garantiza el reconocimiento social a través de un trabajo acorde con sus aspiraciones" (Welti, 2016). Asimismo, afirma que la maternidad continúa siendo un elemento socialmente valorado, a pesar de que la población femenina ha incrementado su nivel de escolaridad y su participación en las actividades económicas y políticas.

Algunos especialistas consideran que el embarazo temprano puede tener como consecuencias el aumento de la feminización de la pobreza en términos de ingresos, así como también en términos de salud y educación; en general, un embarazo adolescente conduce al abandono de los estudios, lo que reduce las posibilidades de conseguir un buen empleo y, por tanto, de mejorar las condiciones de vida, de manera que un proceso educativo incompleto puede repercutir en el resto de la vida de las jóvenes madres y de sus familias (Climent, 2003; FMI, 2013; Cárdenas, De Hoyos y Székely, 2015; Gasparini y Marchionni, 2015).

No existe una definición de adolescencia aceptada internacionalmente. La Organización de las Naciones Unidasy otros organismos establecen quela adolescencia incluye el periodo de vida comprendido entre los 10 y los 19 años, que puede dividirse en adolescencia temprana, de los 10 a los 14 años, y tardía, de los 15 a los 19 años (OMS, s.f.; Dulanto, 2000; Breinbauer y Maddaleno, 2005; UNFPA, 2013):

La adolescencia es un periodo de preparación para la edad adulta durante el cual se producen varias experiencias de desarrollo de suma importancia. Más allá de la maduración física y sexual, esas experiencias incluyen la transición hacia la independencia social y económica, el desarrollo de la identidad, la adquisición de las aptitudes necesarias para establecer relaciones de adulto y asumir funciones adultas y la capacidad de razonamiento abstracto. Aunque la adolescencia es sinónimo de crecimiento excepcional y gran potencial, constituye también una etapa de riesgos considerables, durante la cual el contexto social puede tener una influencia determinante (OMS, s.f.) 
Como se aprecia en la definición, la adolescencia es considerada como un período de tránsito en el desarrollo biológico y social del individuo, pero no se precisa que es una fase clave en la vida de las personas cuya especificidad debe ser reconocida y considerada tanto en términos teóricos, como de política pública. ${ }^{3}$

La Organización Mundial de la Salud señala que en el mundo anualmente dieciséis millones de mujeres menores de 18 años tienen hijos, es decir, una de cada cinco mujeres tiene un hijo antes de cumplir los 18 años. Esta organización calcula que el 95\% de los partos ocurre en países en vías de desarrollo, y que en las regiones más pobres una de cada tres mujeres es madre en la adolescencia, por lo que su juventud queda interrumpida, lo que tiene correlación con la pobreza (OMS, 2011). Una de las preocupaciones más importantes señalada por el Fondo de Población de Naciones Unidas (UNFPA) es el hecho de que, de los dieciséis millones de partos que ocurren en el mundo cada año, casi dos millones se presenta en niñas menores de 15 años; el Fondo identifica América Latina y el Caribe como la región del mundo con mayor aumento en el número de partos en niñas menores de 15 años, e incluso prevé que este aumento continúe hasta el año 2030. Esto representa una clara situación de embarazos no deseados, muchos de los cuales se originan en contextos de abuso de menores, y sobre todo de falta de responsabilidad de los progenitores masculinos frente a la pareja y la paternidad (UNFPA, 2013 y 2014).

En general, las estadísticas y estimaciones comparables disponibles sobre embarazos o partos en adolescentes se enfocan principalmente en la cohorte de 15 a 19 años de edad. En México, las estadísticas oficiales disponibles consideran la cohorte de 12 a 19 años, lo que significa que hay poca información sobre el segmento de población incluido entre los 10 y los 14 años.

\section{La fecundidad adolescente en Chiapas}

Diariamente mil niñas y adolescentes de entre 10 y 19 años de edad se convierten en madres según los datos del Subsistema de Información sobre Nacimientos (SINAC), de acuerdo con los cuales en 2014 hubo en el país 374000 nacidos vivos de madres de entre 10 y 19 años de edad, de los cuales 6000 nacieron de niñas menores de 14 años (Secretaría de Salud, 2014). Esto refleja la existencia de un serio problema por atender. En cifras del SINAC, las características sociodemográficas de las madres adolescentes en México muestran que el $84 \%$ de las niñas que registraron un hijo nacido vivo tenía 14 años; en cuanto a nivel educativo, el $35 \%$ tenía secundaria incompleta, el 23\% primaria completa y el $17 \%$ primaria incompleta; el $72 \%$ de las jóvenes vivían en unión libre o estaban casadas; el 64\% estaba afiliada al Seguro Popular y el 20\% no contaba con ninguna afiliación. La tasa de fecundidad adolescente en mujeres de 15 a 19 años hablantes de una lengua indígena era de 96.7 por mil mujeres, mientras que la tasa de mujeres no indígenas era de 68 por mil (Secretaría de Salud, 2014). El INEGI afirmaba en 2015 que el 12.7\% de las adolescentes en México había tenido al menos un hijo nacido vivo (INEGI, 2015). Considerando que México es un país con grandes diferencias sociales y multiculturales, el estudio de las causas y efectos del embarazo adolescente debe tomar en cuenta los contextos particulares, y debe abordarse desde visiones integrales y multidisciplinarias.

Históricamente, la mayor cantidad de embarazos en mujeres adolescentes se ha presentado en los estados de Chiapas, Oaxaca, Guerrero y Puebla. De acuerdo con la Encuesta Nacional de la Dinámica Demográfica (ENADID) 2014, Chiapas ocupa el tercer lugar en número de embarazos en menores de 19 años; la misma encuesta señala que la tasa de fecundidad adolescente pasó de 87.63 por cada mil en 2009, a 93.25 en 2014, por lo que es un estado con un importante problema de fecundidad en la adolescencia. Por su parte, en la "Estrategia nacional de prevención del embarazo en adolescentes" (ENAPEA) se indica que en 2015 el promedio anual de embarazos tempranos era de 884 (CONAPO, 2015a; INEGI, 2014 y 2015; ENAPEA, 2015).

Adicionalmente, la ENADID 2009-2014 indica que en México, la tasa de fecundidad adolescente (TFA) 
de 15 a 19 años es de 69.5 nacimientos por mil mujeres, mientras que la del Estado de Chiapas es del 89.1, por mil (INEGI, 2014), a nivel mundial la tasa es de 44.7 por cada mil mujeres (Banco Mundial, 2017a). Por otro lado, los datos de la Encuesta Intercensal 2015 señalan que la tasa global de fecundidad (TGF) en México entre mujeres indígenas es de 3.1 hijos por mujer, mayor con respecto a la general, que es de 2.3 (INEGI, 2015), mientras que a nivel mundial la tasa registrada fue de 2.4 en el año 2015 según datos del Banco Mundial (2017b).

A esta situación ha de agregarse que Chiapas es la entidad con uno de los índices de marginación más altos; que la mediana de edad del estado se ubica en 21.2 años; que tiene una proporción del $322.48 \%$ de niños y adolescentes de entre 10 y 19 años; que la proporción de éstos que ya ha iniciado su vida sexual es el 20.2\% mayor que en la década pasada, y que la entidad ocupa el primer lugar con menor uso de anticonceptivos en la primera relación afectiva (ENSANUT, 2012; CONAPO, 2015a y 2015b).

Sibien es clara la condición del embarazo en menores de 19 años en Chiapas, también es cierto que no existe información ni estadísticas sobre el comportamiento del fenómeno a nivel municipal dado que el territorio estatal es diverso geográfica, cultural, económica y socialmente. Tampoco existe información focalizada sobre el comportamiento del embarazo de niñas de entre 10 y 14 años, ni se ha analizado el fenómeno contrastándolo con el grado de marginación y la condición de indigenismo a nivel municipal. Esta información es fundamental para diseñar intervenciones que tengan mayor impacto en la reducción del fenómeno.

La presente investigación parte de la hipótesis de que las tasas específicas de fecundidad en mujeres adolescentes son superiores en poblaciones indígenas ${ }^{4}$ y en grupos con mayores desventajas sociales o educativas, y que estas características configuran patrones específicos de distribución espacial que reflejan mayores tasas de fecundidad adolescente en los municipios con alta o muy alta marginación, donde la población mayoritariamente es indígena o presenta bajos niveles de escolaridad.

\section{Fuentes de información y datos}

La estimación de las tasas de fecundidad de los municipios de Chiapas se realizó con datos obtenidos de la Encuesta Intercensal 2015 del Instituto Nacional de Estadística y Geografía (INEGI, 2015), de donde además se compiló información sobre población total, población indígena y población analfabeta a nivel estatal y municipal. La encuesta garantiza representatividad a nivel municipal, en especial en municipios marginados, indígenas y con un alto porcentaje de localidades rurales dispersas, sobre temas como: población total, estructura por edad y sexo, fecundidad, mortalidad, migración, movilidad, etnicidad, acceso a servicios de salud y educación, trabajo no remunerado y características económicas. Los datos de la Encuesta Intercensal 2015 permitieron estimar las tasas específicas de fecundidad (TEF) a nivel municipal para Chiapas: la tasa de fecundidad adolescente temprana en el grupo de entre 10 y 14 años, la tasa de fecundidad adolescente tardía en el grupo de entre 15 y 19 años y la tasa de fecundidad adolescente general en el grupo de entre 10 y 19 años.

Los datos referentes a los niveles de marginación municipal fueron tomados del Índice de Marginación por Entidad Federativa y Municipio 2015 del Consejo Nacional de Población (CONAPO), institución oficial que estima y publica las cifras sobre marginación en México. Es importante señalar que el índice de marginación es un indicador de tipo multidimensional que resumeinformación de nueve variables agrupadas en cuatro dimensiones: educación, vivienda, distribución espacial de la población e ingresos monetarios. Elíndice de marginación mide la intensidad de la segregación económica, social y espacial a través de variables como el analfabetismo, la educación primaria incompleta y la carencia de servicios en la vivienda, como drenaje, excusado, energía eléctrica, agua entubada, piso de tierra y hacinamiento. La dimensión de marginación económica se mide como el porcentaje de población con ingresos de hasta dos salarios mínimos, y la segregación espacial se estima cuantificando el porcentaje de población que reside en localidades menores de cinco mil habitantes (CONAPO, 2015a). 


\section{Estimación de la fecundidad adolescente municipal}

Con la finalidad de contar con estimaciones robustas sobre el comportamiento de las tasas de fecundidad adolescente, fue necesario reconstruir la historia de la fecundidad general en Chiapas desde el año 1970, para lo que se utilizó la metodología de estimación propuesta por el UNFPA en el documento "Direct estimation of fertility from survey data containing birth histories" (UNFPA, s.f.).

La gráfica 1 muestra las estimaciones de fecundidad por edad y año calendario ( 1 de enero al 31 de diciembre). Como se puede apreciar en esta gráfica, el comportamiento de la fecundidad en los años setenta (curvas de colores grises claros) presentaba un pico de alta fecundidad después de los 30 años, la edad de inicio de fecundidad era tardía, posterior a los 20 años, y la fecundidad total era bastante elevada. En las décadas de los ochenta y noventa (curvas centrales) se observó una importante reducción en los niveles de fecundidad general y un desplazamiento en el pico de la fecundidad, que se ubicaba alrededor de los 30 años de edad; adicionalmente la fecundidad en adolescentes comenzó a ser importante. Después del año 2000 (curvas en tono gris claro), se observa un repunte sostenido de los niveles de fecundidad, con el inicio de la etapa reproductiva desde los 15 años, y la curva de fecundidad alcanza su máximo alrededor de los 30 años; es importante notar que en este periodo la fecundidad adolescente ha crecido en importancia, en especial en mujeres menores de 17 años.

\section{Análisis de los datos}

Uno de los métodos estadísticos más utilizados para medir el grado de asociación lineal entre dos variables cuantitativas es el coeficiente de correlación de Pearson, el cual se define dividiendo la covarianza por el producto de las desviaciones estándar de ambas variables, de modo que:

$$
r_{x y}=\frac{s_{x y}}{s_{x} \cdot s_{y}}=\frac{\frac{1}{n} \sum_{i}\left(x_{i}-\bar{x}\right)^{2}}{\sqrt{\frac{1}{n} \sum_{i}\left(x_{i}-\bar{x}\right)^{2}} \cdot \sqrt{\frac{1}{n} \sum_{i}\left(y_{i}-\bar{y}\right)^{2}}}
$$

Donde $r_{x y}$ toma valores entre - 1 y 1 . Un valor cercano a cero indica la ausencia de asociación lineal entre las variables, y valores cercanos a $10-1$ indican una correlación positiva o negativa, respectivamente, entre las variables.

En la gráfica 2 se presenta el coeficiente de Pearson para: la tasa específica de fecundidad de 10 a 19 años (TEF 10-19); el índice de marginación (IM); la población femenina hablante de lengua indígena (HLI); la escolaridad femenina media (escmedia), y el porcentaje de analfabetismo femenino (analfabeto).

Como se puede apreciar en la gráfica 2, existe un nivel de correlación alto (mayor a |0.5|) entre todas las variables presentadas. Sin embargo, debe señalarse que el coeficiente de Pearson cuantifica el grado de asociación estadística entre dos variables, pero no considera el efecto espacial, es decir, no toma en cuenta el efecto de la distancia entre municipios. Por ejemplo, se observa que la tasa de fecundidad en menores de 19 años tiene una correlación positiva con el índice de marginación (0.67); no obstante, cuando se analiza el efecto espacial, este nivel de correlación se diluye, situación que no ocurre con la condición de hablante de lengua indígena.

\section{Análisis exploratorio de datos espaciales}

Adicional al análisis de correlación de datos estadísticos, el análisis exploratorio de datos espaciales (AEDA) es un método para el estudio de la correlación entre una variable y el espacio geográfico sobre el que se distribuye, lo que se conoce como autocorrelación espacial. Con el AEDA se busca comprobar la existencia de correlaciones espaciales, por lo que es necesario definir relaciones de vecindad entre unidades espaciales. En el caso de estudio, las unidades espaciales están conformadas por municipios, mientras que el criterio de vecindad se definió a partir de la distancia, de modo que dos municipios se consideran vecinos si la distancia entre sus cabeceras municipales es menor o igual a 54.6 kilómetros — criterio conocido como de vecino más cercano, el cual se define a través de la contigüidad por distancias medidas entre centroides, dentro de 
una banda establecida como mínima, considerándose vecinas dos unidades espaciales cuando están situadas dentro de la banda-

Una vez identificadas las vecindades, se definió la matriz de interacciones espaciales (también conocida como matriz de contigüidades), donde dij = 1, si los municipios $\mathrm{i}$, $\mathrm{j}$ son vecinos, $\mathrm{y}$ dij $=0$ en caso contrario; además, dii = 0 por convención. Es importante señalar que la matriz de interacciones espaciales es una matriz binaria y simétrica, es decir, dij = dji, para todo i, j (Chasco, 2003). La matriz de interacciones permite establecer la existencia, o no, de una relación de vecindad entre un conjunto de municipios; sin embargo, no permite cuantificar la importancia de la relación, para lo cual se define la matriz de pesos espaciales.

La matriz de pesos espaciales se define a partir de un conjunto de ponderadores ajustados a cada fenómeno y a las unidades espaciales estudiadas, lo que otorga robustez a la estructura de dependencias espaciales. La matriz W de pesos espaciales se define como una matriz simétrica cuyos elementos [wij] toman el valor de cero en ausencia de autocorrelación espacial entre los municipios i, j. Cuando wij $\neq 0$ existe una interacción espacial que recoge el efecto del municipio i sobre el municipio j a través del peso wij. En el caso de estudio, la matriz de pesos espaciales se definió a partir de la estandarización de los renglones de la matriz de contactos, de forma que el elemento i de cada renglón j se dividió por la suma de los contactos del renglón:

$$
w_{i j}=\frac{\delta_{i j}}{\sum_{j} \delta_{i j}}
$$

De esta forma se garantiza que la suma de los pesos de cada renglón sea igual a la unidad, y la suma de todos los renglones de la matriz sea igual al total de municipios, es decir, al tamaño de la muestra.

\section{Índice de Moran}

En general, la presencia de patrones espaciales suele captarse a partir de la observación de coincidencias en un mapa sobre el que se dibuja la distribución de la variable analizada; no obstante, los patrones detectados de esta manera carecen de un análisis estadístico objetivo que permita replicar o cuantificar adecuadamente las asociaciones detectadas, por lo que, para detectar la presencia de correlaciones entre una variable y su distribución espacial, se recomienda el uso de estadísticos diseñados específicamente para tal fin (Anselin, 1995); estos estadísticos son conocidos como índices de autocorrelación espacial.

Los estadísticos de autocorrelación espacial tienen la capacidad de resumir en un único índice la dependencia detectada entre una variable y la forma en que se distribuye en el espacio, con lo que es posible detectar agrupaciones o clusters, los cuales pueden ser clasificados como locales o globales. En general cada estadístico cuenta con ambas versiones.

Uno de los estadísticos más populares para medir la autocorrelación espacial es el índice de Moran, que en su versión global es útil para cuantificar la distancia ponderada en términos de desviaciones de las unidades espaciales $(i, j)$ respecto de la media, ponderada por la matriz de pesos espaciales, wij (Cliff y Ord, 1981). Cuando se utiliza una matriz de pesos espaciales estandarizada por renglones, el estadístico de Moran puede calcularse como:

$$
I=\frac{\sum_{i} \sum_{j} w_{i j}\left(y_{i}-\bar{y}\right)\left(y_{j}-\bar{y}\right)}{\sum_{i}\left(y_{i}-\bar{y}\right)^{2}}
$$

Es importante señalar que la media teórica del índice de Moran está dada por:

$$
E[I]=\frac{-1}{N-1}
$$

por lo que su valor esperado está en función del tamaño de la muestra $(\mathrm{N})$, es negativo y tiende asintóticamente a cero conforme $\mathrm{N}$ aumenta.

El índice de Moran toma valores entre $-1 \mathrm{y}+1$. Valores de autocorrelación espacial positiva indican la presencia de un conglomerado de unidades espaciales con valores de análisis similares, lo que ocurre cuando el índice de Moran es mayor que su valor esperado. Valores de autocorrelación espacial negativa indican la presencia de un conglomerado espacial con valores disímiles, lo que se presenta cuando el índice toma valores inferiores a 
su media. Finalmente, valores cercanos a cero indican la ausencia de autocorrelación espacial.

El índice de Moran I es un estadístico cuya distribución, una vez estandarizada, toma la forma de una normal estándar, de modo que:

$$
z=\frac{I-E[I]}{\sqrt{\operatorname{var}(I)}} \sim N(0,1)
$$

Por ello, valores de $z$ significativamente distintos de cero serán evidencia de la presencia de autocorrelación espacial, es decir, valores estadísticamente significativos con un $\mathrm{p}$-value inferior a 0.05 , lo que conducirían a rechazar la hipótesis nula de no autocorrelación espacial, con lo que se aceptaría la existencia de patrones espaciales de la variable analizada. La tabla 1 muestra los valores del índice de Moran global para las variables analizadas en el estudio; en general, todas presentan un importante nivel de asociación espacial positiva (ver tabla l).

Un resultado importante, presentado en la tabla 1 , señala que los valores del índice de Moran resultaron ser positivos en todos los casos - además presentaron altos niveles de significancia estadística, $z$ mayor a 2 y un p-value menor a 0.05 en todos los casos-, lo que indica que existe evidencia que muestra la presencia de autocorrelación espacial positiva en los municipios de Chiapas para las variables: tasa global de fecundidad (TGF), fecundidad adolescente (general 10-19, temprana 10-14 y tardía 15-19), el índice de marginación, la población hablante de lengua indígena, porcentaje de población analfabeta femenina y escolaridad media femenina. Ello significa que los municipios con altos niveles de fecundidad adolescente están rodeados por municipios que a su vez presentan tasas de fecundidad adolescente por encima del promedio estatal y viceversa.

Si bien el índice de Moran global muestra evidencia de la existencia de conglomerados, no es útil para identificarlos específicamente, razón por la que se desarrolló la versión local del mismo. Elíndice de Moran local permite identificar el tamaño y localización de los conglomerados espaciales, con lo que es posible crear diferentes diagramas y mapas que permitan visualizar espacialmente la distribución de la variable analizada.

\section{Análisis espacial de la fecundidad municipal en Chiapas}

Las medidas de autocorrelación locales, denominadas también indicadores Locales de Asociación Espacial (LISA) (Anselin, 1995), permiten detectar la presencia de regiones de dependencia espacial dentro de un espacio geográfico, es decir, permiten identificar clusters que configuran espacios locales. Por ejemplo, el diagrama de dispersión de Moran o scatterplot (Anselin, 1988), uno de los indicadores LISA más utilizados, permite visualizar el nivel de correlación lineal de cada unidad espacial en función de la variable analizada y su retardo espacial. ${ }^{5}$ Cada punto del diagrama representa una unidad espacial. En el eje X se representan las desviaciones respecto de la media de la variable analizada. En el eje Y se presenta el retardo espacial de la variable, y la pendiente de la recta representa el valor del índice de Moran global, por lo que una mayor inclinación implica una mayor autocorrelación espacial.

El diagrama de Moran divide la gráfica en cuatro cuadrantes de asociación espacial; en los cuadrantes Iy III se ubican las unidades espaciales con autocorrelaciones espaciales positivas y negativas, respectivamente, y en los cuadrantes II y IV se encuentran: las unidades espaciales con autocorrelaciones espaciales de valores altos rodeados de unidades con valores bajos en el cuadrante II, y viceversa en el cuadrante IV (ver gráfica 3).

Por ejemplo, en la gráfica 3 puede verse el diagrama de dispersión de Moran para la escolaridad media femenina, donde es notoria la inclinación de la recta, lo que confirma una vez más los altos niveles de autocorrelación espacial positiva de las variables analizadas (ver mapas l.a. a l.d.).

Es importante señalar que cada punto en la diagrama de Moran representa un municipio y que los municipios que se encuentran agrupados en los distintos cuadrantes conforman conglomerados espaciales de la variable respectiva, los cuales pueden ser observados con mayor claridad en un mapa de Moran.

El mapa de Moran muestra la formación de conglomerados para cada una de las variables analizadas (ver mapas l.a. a 1.d.). Los conglomerados 
se forman al agrupar los municipios para cada uno de los cuatro cuadrantes, tal y como se muestra en el diagrama de Moran, de modo que en los mapas l.a. a l.d. se identifican los clusters, que agrupan municipios con distintas combinaciones de valores altos o bajos de la variable analizada, con sus respectivas combinaciones.

En general, los mapas de Moran identifican cinco tipos de conglomerados: 1) High-High: unidades territoriales con valores por encima del promedio, rodeadas por unidades con valores superiores a la media, llamados conglomerados calientes o hot spots y se representan en gris oscuro; 2) Low-Low: conglomerados formados por unidades espaciales cuyo valor de análisis es inferior al promedio, que están rodeados por unidades con valores por debajo de la media, son llamados conglomerados fríos o cold spots, y en general se dibujan en negro; 3) Low-High son conglomerados formados por unidades espaciales con un valor por debajo de la media, que están rodeados por unidades con valores sobre la media, y suelen dibujarse de gris rayado; 4) High-Low: presenta conglomerados de unidades espaciales con valores por arriba del promedio rodeados por unidades espaciales que presentan valores por debajo de la media, y suelen colorearse en gris claro; 5) No data: son las unidades espaciales que no presentan relación significativa con sus unidades vecinas respecto del valor de la variable de interés analizada.

En el mapa l.a pueden observarse los cinco tipos de conglomerados para la variable tasa global de fecundidad (TGF) respecto de los 118 municipios de Chiapas durante el año 2015, de acuerdo con la clasificación realizada por el índice de Moran local. El mapa de autocorrelación espacial para la TGF hace posible identificar un conglomerado caliente (gris oscuro), es decir, un conjunto de municipios con altos niveles de fecundidad rodeados de municipios con niveles de fecundidad por encima de la media estatal; estos se encuentran ubicados en la zona de Los Altos y son los municipios de Larráinzar, Chamula y Chenalhó, entre otros; además se distinguen dos conglomerados fríos (negros), o municipios donde se observan niveles relativamente bajos de fecundidad, que a su vez están rodeados de municipios con niveles de fecundidad por debajo del promedio estatal, los cuales se encuentran ubicados en la zona Istmo-Costa, en municipios como Arriaga, Tonalá, Villaflores y Jiquipilas, y en la zona del Soconusco en los municipios de Tapachula, Mazatán y Huehuetán, entre otros.

El comportamiento espacial de la fecundidad adolescente (10-19) en los municipios de Chiapas es muy similar al presentado por la TGF y la tasa de fecundidad adolescente tardía (15-19), por lo que se decidió omitir su explicación. No obstante, el mapa l.b., referente al comportamiento espacial de las tasas de fecundidad adolescente temprana (10-14), presenta un conglomerado rojo en la zona fronteriza con Guatemala, en los municipios de Marqués de Comillas, Benemérito de las Américas y Maravilla Tenejapa, pertenecientes a la región Selva. Por la ubicación de los municipios y por la edad de las mujeres, los embarazos pudieran ser resultado de condiciones de explotación y trata de menores, especialmente de mujeres indígenas, migrantes o que se encuentran en condiciones de alta vulnerabilidad.

En los mapas 2.a. a 2.d. se presenta un análisis de la distribución espacial de algunos de los determinantes que se consideraron más importantes para entender el comportamiento espacial de la fecundidad adolescente en el estado de Chiapas. En el mapa 2.a. se puede observar un conglomerado caliente formado por los municipios donde se concentran los mayores niveles de marginación, como Larráinzar, Chamula y Chenalhó, por mencionar algunos. También se observan dos conglomerados fríos, uno en la región Istmo-Costa, conformado por los municipios de Arriaga, Tonalá, Cintalapa y Jiquipilas, y otro en la región del Soconusco formado por los municipios de Tapachula, Huixtla, Mapastepec, entre otros.

La dispersión espacial de la proporción de población indígena municipal de Chiapas durante el año 2015 puede verse en el mapa 2.b., donde se observan dos conglomerado o zonas frías (negro): uno ubicado en la zona del Soconusco, en el municipio de Tapachula, y otro en la zona Istmo-Costa que comprende los municipios de Cintalapa, Arriaga, Tonalá y Jiquipilas. 
Al mismo tiempo se observa un conglomerado caliente (gris oscuro) conformado por los municipios con los mayores niveles de población indígena, que a su vez están rodeados de municipios con altos niveles de población indígena. Este conglomerado se encuentra en las zonas Selva y Altos y se encuentra conformado por los municipios de Larráinzar, Chamula y Chenalhó, entre otros.

Los niveles de escolaridad promedio y analfabetismo femenino se presentan en los mapas 2.c. y 2.d., respectivamente. Es evidente que el conglomerado con los menores niveles de escolaridad parece empalmarse con el conglomerado con las mayores tasas de analfabetismo; sin embargo, el analfabetismo y la escolaridad son conceptos que, aunque pudieran parecer similares, distan mucho de serlo. El analfabetismo se entiende por la incapacidad para leer y escribir un recado, y la escolaridad tiene que ver con el acceso y la permanencia en el sistema educativo, aunque la asistencia al sistema escolar no garantiza la alfabetización ni viceversa. En general, la bibliografía señala que, a mayor escolaridad (Cárdenas, De Hoyos y Székely, 2015; Gasparini y Marchionni, 2015), las mujeres tienden a posponer y reducir su fecundidad, y que las mayores tasas de fecundidad ocurren entre mujeres analfabetas, por lo que es de esperar que la distribución espacial de ambos fenómenos coincida con la distribución de la fecundidad adolescente en los municipios estudiados.

\section{Conclusiones}

Es importante señalar que, si bien el objetivo de la investigación fue identificar la presencia de patrones espaciales de fecundidad adolescente municipal en Chiapas, alcanzarlo implicó el uso de una estrategia basada en el análisis estadístico de datos espaciales, lo que hizo posible la detección de distintos conglomerados de fecundidad adolescente, durante el año 2015. Tales conglomerados reflejan la existencia de zonas donde las tasas de fecundidad adolescente temprana y tardía son mayores; sin embargo, lo preocupante de esta situación resulta de dos hechos: 1) las tasas de fecundidad adolescente en Chiapas, lejos de reducirse, presentan desde el año 2000 una tendencia creciente, y 2) las zonas donde se concentran las mayores tasas de fecundidad adolescente parecen coincidir con los conglomerados con mayores niveles de marginación, analfabetismo femenino, proporción de población indígena y menores niveles de escolaridad promedio entre mujeres.

La identificación de patrones espaciales de fecundidad adolescente municipal se acompañó de la detección de conglomerados para un conjunto de determinantes de la fecundidad adolescente, entre los que se incluyeron: la marginación, la pertenencia a una etnia indígena, la escolaridad promedio femenina y el analfabetismo femenino. Los resultados mostraron la presencia de patrones espaciales para todos los determinantes estudiados, situación que parece implicar la presencia de correlaciones espaciales entre los determinantes analizados y las tasas de fecundidad adolescente en los municipios de Chiapas, con lo que el peso explicativo de los determinantes tendía, además de a una asociación teórica y estadística, a una relación a nivel espacial.

En concordancia con la conclusión anterior, se puede señalar que la posible asociación entre patrones espacialmente correlacionados entre la fecundidad adolescente y los determinantes estudiados reforzaría sin duda la importancia de analizar distintos aspectos socioculturales; especialmente valdría la pena profundizar en las relaciones de género, el papel y el significado de la maternidad y su conceptualización entre diferentes grupos étnicos. Analizar espacialmente un fenómeno implica, desde luego, acercarse a su contexto.

Finalmente, el patrón detectado de concentracióndispersión espacial de fecundidad adolescente en los municipios de Chiapas aportará elementos importantes para el diseño de estrategias dirigidas a los municipios que presentan mayores niveles de fecundidad adolescente y que a su vez se encuentran en los conglomerados donde se observó una mayor proporción de población indígena, y de población con mayores niveles de marginación y analfabetismo, y con menores niveles de escolaridad promedio. 


\section{Notas}

${ }^{1}$ Ver: Szasz (1998), Schmukler (1998), Stern y García (1995, 2001), Stern (2004) y Tuñón (2006.

${ }^{2}$ Más de dos millones de jóvenes de entre 10 y 19 años viven con VIH; alrededor de una de cada siete nuevas infecciones se produce durante la adolescencia (UNFPA, 2014).

3 "Los adolescentes y jóvenes merecen ser visibilizados poniendo de relieve sus acciones positivas, no como un problema. Hay que ser capaces de ver sus fortalezas, más aún si se considera que ésta constituye la etapa de la vida en que las personas están consolidando sus identidades, y se espera que logren la construcción identitaria más positiva posible. La adolescencia es la etapa de la vida en que se producen los procesos de maduración biológica, psíquica y social de un individuo, que le permiten alcanzar su incorporación en forma plena a su cultura y sociedad" (Rodríguez, 2011).

${ }^{4}$ En general, los pueblos indígenas de Chiapas son aquellos grupos de población que descienden de los que habitaban el territorio antes de la llegada de los españoles en el siglo XVI, que poseen una lengua y cultura propias, y que comparten formas de vida y cosmovisiones particulares diferenciadas de la cultura occidental (Bello y Rangel, 2002).

${ }^{5}$ El retardo espacial es un concepto que cuantifica la relación que mantiene cada unidad espacial con sus unidades vecinas. Se define en términos del grado de semejanza que alcanzan los valores promedio ponderados de la variable analizada (de la unidad estudiada) respecto de sus unidades vecinas.

\section{Referencias}

Anselin, L. (1988). Spatial economitrics: Methods and models. Países Bajos: Kluwer Academic Publisher.

Anselin, L. (1995). "Local Indicators of Spatial AssociationsLISA". En Geographical Analysis, 27: 93-115.

Banco Mundial (2017a). "Tasa de fecundidad en adolescentes (nacimientos por cada 1000 mujeres entre 15 y 19 años de edad". Disponible en: http://
datos.bancomundial.org/indicador/SP.ADO.TFRT (consultado el 9 de junio de 2017)

Banco Mundial (2017b). "Tasa global de fecundidad (nacimientos por mujer)". Disponible en: http:// datos.bancomundial.org/indicador/SP.DYN.TFRT.IN (consultado el 9 de junio de 2017).

Breinbauer, Cecilia y Matilde Maddaleno (2005). Youth, Choice and Change. Promoting healthy behaviors in adolescents. Washington, D.C.: PAHO.

Bello, A. y M. Rangel (2002). "La equidad y la exclusión de los pueblos indígenas y afrodescendientes en América Latina y el Caribe". En Revista CEPAL, 76: 39-54.

Cárdenas, Mauricio, Rafael de Hoyos y Miguel Székely (2015). "Out-of-School and Out-of-Work Youth in Latin America: A Persistent Problem in a Decade of Prosperity". En Economía, 16(1): 1-40

Cliff, A.D. y J.K. Ord. (1981). Spatial Processes: Models and Applications. Londres: Pion Limited.

Climent, G. (2003). "La maternidad adolescente, una expresión de la cuestión social. El interjuego entre la exclusión social, la construcción de la subjetividad y las políticas públicas". En Revista Argentina de Sociología, l(1): 77-93.

CONAPO (2015a). Salud sexual y reproductiva. Chiapas. México: Consejo Nacional de Población. Disponible en:http://www.conapo.gob.mx/work/models/CONAPO/ Libros_Salud/CS/index.html\#/3/zoomed.

CONAPO (2015b). Datos Abiertos del Índice de Marginación. Índice de marginación por entidad federativa 1990-2015. México: Consejo Nacional de Población. Disponible en: http://www.conapo.gob.mx/es/CONAPO/Datos_ Abiertos_del_Indice_de_Marginacion.

Chasco, C. (2003). Econometría espacial aplicada a la predicción-extrapolación de datos microterritoriales. Madrid: Consejería de Economía e Innovación Tecnológica, Comunidad de Madrid.

Dulanto, E. (2000). El adolescente. México: McGraw-Hill Interamericana.

ENAPEA (2015). "Estrategia nacional para la prevención del embarazo en adolescentes". México: Gobierno de la República. Disponible en: http://www.conapo. gob.mx/es/CONAPO/ESTRATEGIA_NACIONAL_ 
PARA_LA_PREVENCION_DEL_EMBARAZO_EN_ ADOLESCENTES (consultado el 6 de mayo de 2016).

ENSANUT (2012). "Encuesta nacional de salud y nutrición 2012. Resultados Nacionales". Cuernavaca, México: Instituto Nacional de Salud Pública.

FMI (2013). Women, Work, and the Economy: Macroeconomic Gains from Gender Equity. Washington, D.C.: FMI.

Gasparini, Leonardo y Mariana Marchionni (eds.) (2015). Bridging Gender Gaps? The Rise and Deceleration of Female Labor Force Participation in Latin America. La Plata, Argentina: CEDLAS.

Gómez de León J. y D. Hernández (2002). "Pobreza y métodos anticonceptivos en México rural". En Cecilia Rabell y María Eugenia Cosío-Zavala (comps.), La fecundidad en condiciones de pobreza. Una visión internacional. México: Instituto de Investigaciones Sociales-UNAM, pp. 213-229.

INEGI (2014). Encuesta nacional de la dinámica demográfica 2014 (ENADID). México: INEGI.

INEGI (2015). Encuesta intercensal 2015. Síntesis metodológica y conceptual. México: INEGI.

OMS (s.f.). "Salud, de la madre, el recién nacido, del niño y del adolescente". Organización Mundial de la Salud. Disponible en: http://www.who.int/maternal_child_ adolescent/topics/adolescence/dev/es/ (consultado el 10 de julio de 2016).

OMS (2011). "Prevenir el embarazo precoz y los resultados reproductivos adversos en adolescentes en los países en desarrollo: las evidencias". Organización Mundial de la Salud. Disponible en:http://www.who.int/maternal child_adolescent/documents/preventing_early_ pregnancy/es/ (consultado el 10 de julio 2016).

Rodríguez Vignoli, Jorge (2011). La reproducción en la adolescencia y sus desigualdades en América Latina. Introducción al análisis demográfico, con énfasis en el uso de microdatos censales de la ronda de 2010. Santiago de Chile: CEPAL. Disponible en:http://www2. congreso.gob.pe/sicr/cendocbib/con4_uibd.nsf/ 91C5FAF13D339E2505257D31005F5820/\$FILE/ Lareproduccionenlaadolescencia.pdf.

Secretaría de Salud (2014). Subsistema de Información sobre Nacimientos SINAC Informe 2013-2014. México:
Secretaría de Salud. Disponible en: http://www.dgis. salud.gob.mx/descargas/pdf/Libro_SINAC_version_ digital.pdf.

Stern, Claudio y Elizabeth García (1995). "Embarazo adolescente. Significado e implicaciones para distintos sectores sociales". En Demos, 8: 11-12.

Stern, Claudio y Elizabeth García (2001). "Hacia un nuevo enfoque en el campo del embarazo adolescente". En Claudio Stern y Juan Guillermo Figueroa (coords.), Sexualidad y salud reproductiva. Avances y retos para la investigación. México: El Colegio de México, pp. 331-364.

Stern, Claudio (2004). "Vulnerabilidad social y embarazo adolescente en México”. En Papeles de Población, 10(39), enero-marzo: 129-158.

Szasz, I. (1998). "Sexualidad y género: algunas experiencias de investigación en México". En Debate Feminista, 9(18), octubre: 77-104.

Schmukler, Beatriz (coord.) (1998). Familias y relaciones de género en transformación. Cambios transcendentales en América Latina y el Caribe. México: Population Council, Edamex.

Tuñón, Esperanza (2006). "Embarazo en adolescentes del sur-sureste de México". En Papeles de Población, 12(48), abril-junio: 141-154.

UNFPA (2013). Maternidad en la niñez. Enfrentar el reto del embarazo en adolescentes. Estado de la población mundial 2013. Nueva York: Fondo de Población de las Naciones Unidas.

UNFPA (2014). Estado de la población mundial 2014. El poder de 1800 millones. Los adolescentes, los jóvenes y la transformación del futuro. Nueva York: Fondo de Población de las Naciones Unidas.

UNFPA (s.f.). Direct estimation of fertility from survey data containin gbirth histories. Nueva York: Fondo de Población de Naciones Unidas. Disponible en: http:// demographicestimation.iussp.org/content/directestimation-fertility-survey-data-containing-birth-histories.

Welti, Carlos (2016). "En México, cada año 400 mil nacimientosdemadres adolescentes". EnElSoldeMéxico, $1 l$ de mayo. Disponible en: http://www.elsoldemexico. com.mx/mexico/220060-en-mexico-cada-ano-400mil-nacimientos-de-madres-adolescentes. 
Gráfica 1. Tasas específicas de fecundidad Chiapas, 1970 a 2015

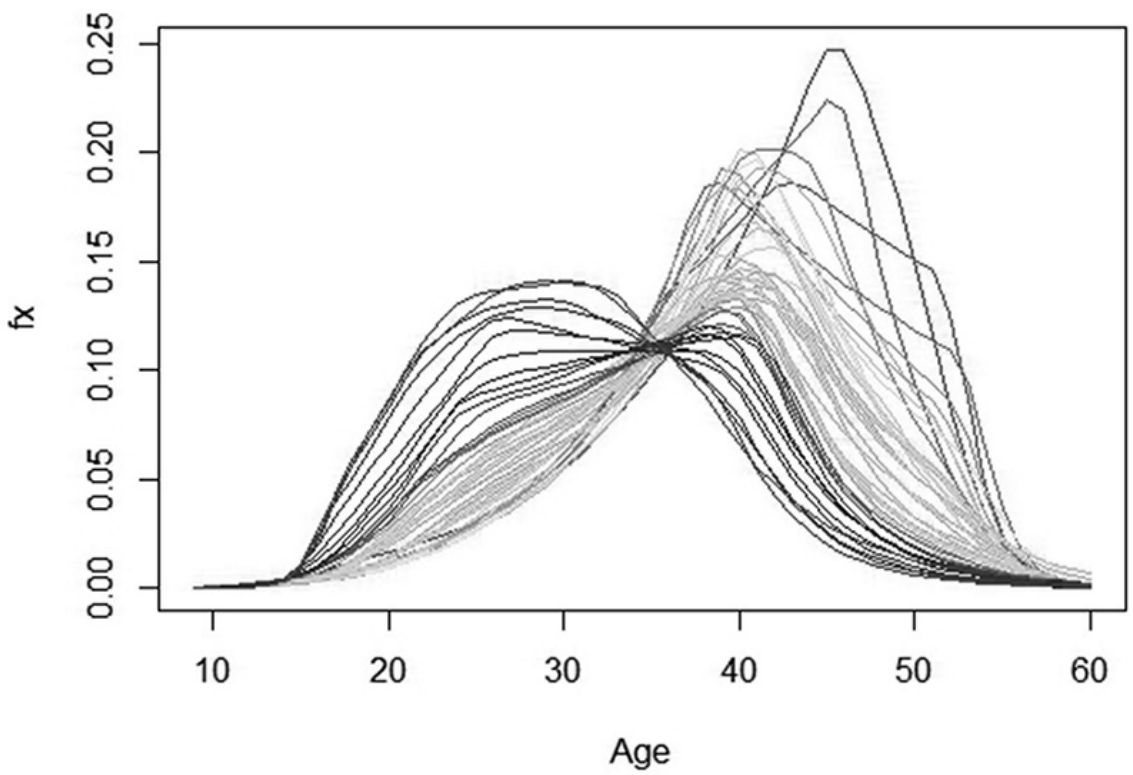

Fuente: elaboración propia con datos de la encuesta intercensal INEGI 2015.

Gráfica 2. Coeficiente de correlación de Pearson

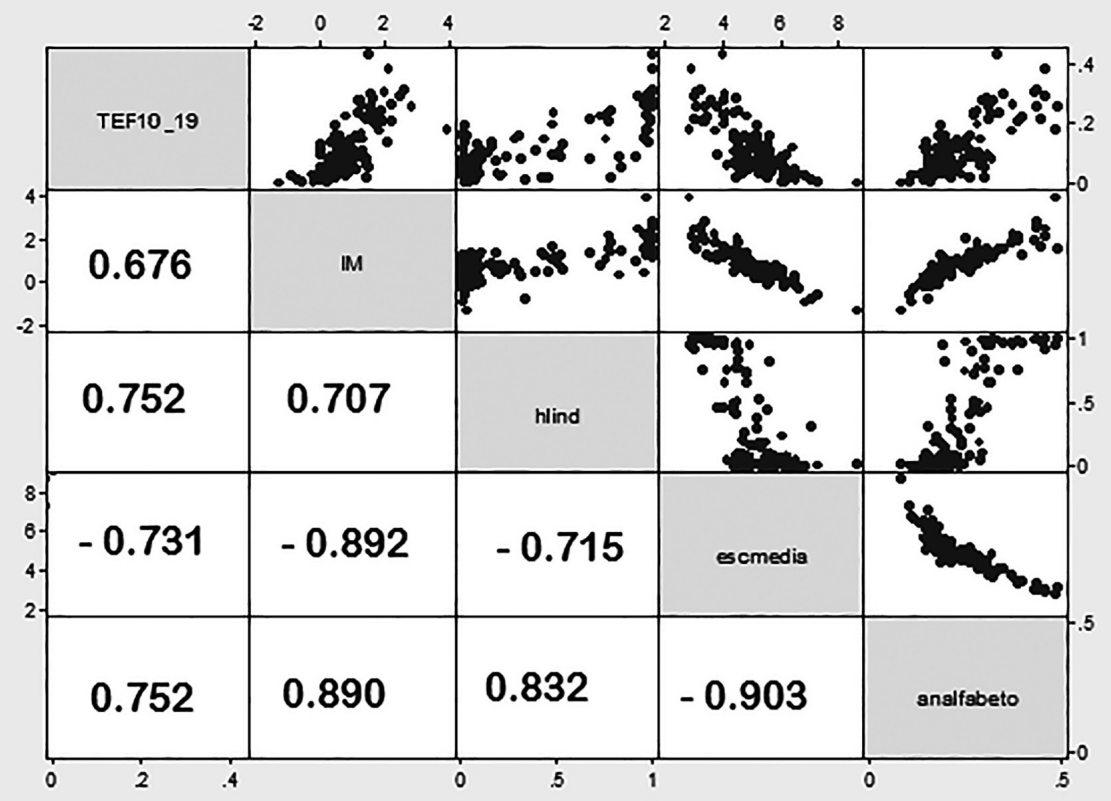

Fuente: elaboración propia con datos de la encuesta intercensal INEGI 2015, de CONAPO 2015. 
Gráfica 3. Diagrama de dispersión de Moran, escolaridad media

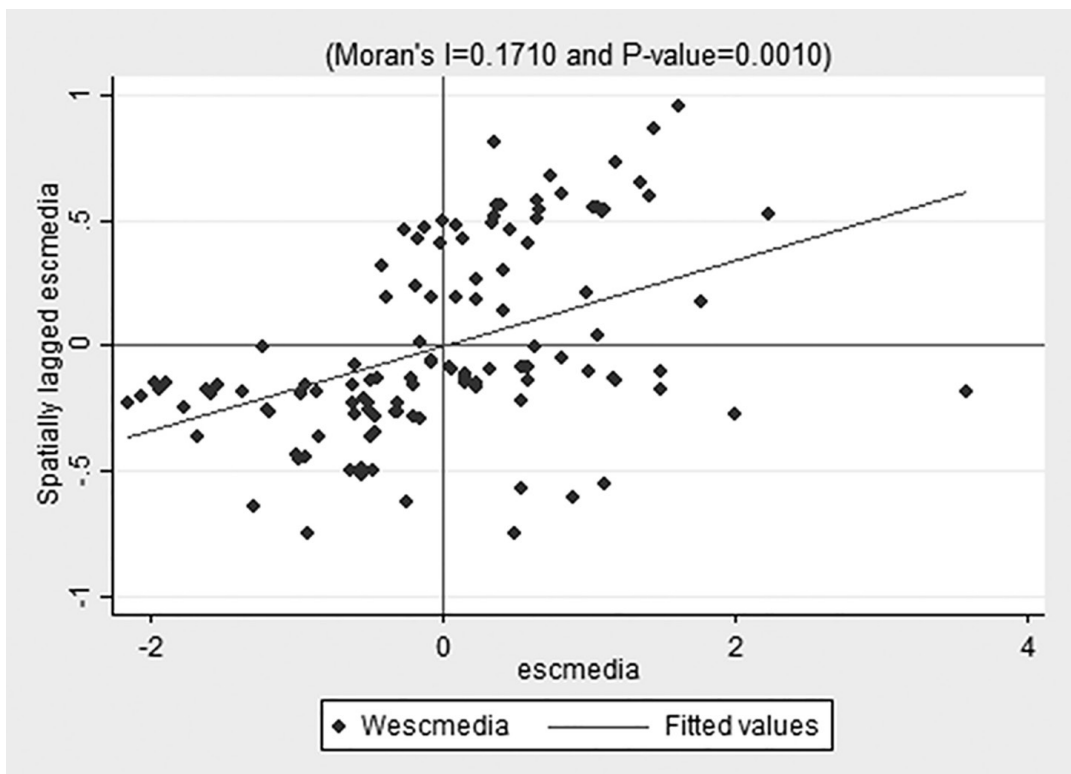

Fuente: elaboración propia con datos de la encuesta intercensal INEGI 2015, de CONAPO 2015.

Tabla 1. índice de Moran

\begin{tabular}{cccc}
\hline Variables & I - moran & $\mathrm{Z}$ & p-value \\
\hline TGF & 0.133 & 7.357 & 0.000 \\
TEF9_14 & 0.067 & 4.111 & 0.000 \\
TEF15_19 & 0.136 & 7.506 & 0.000 \\
TEF_9_19 & 0.134 & 7.419 & 0.000 \\
IM & 0.140 & 7.807 & 0.000 \\
HLI & 0.328 & 17.44 & 0.000 \\
Analfabeto & 0.240 & 12.983 & 0.000 \\
Escmedia & 0.171 & 9.371 & 0.000 \\
\hline
\end{tabular}

Fuente: elaboración propia con datos del INEGI 2015. 
Mapas 1.a. a 1.d. Autocorrelación espacial de la fecundidad en Chiapas

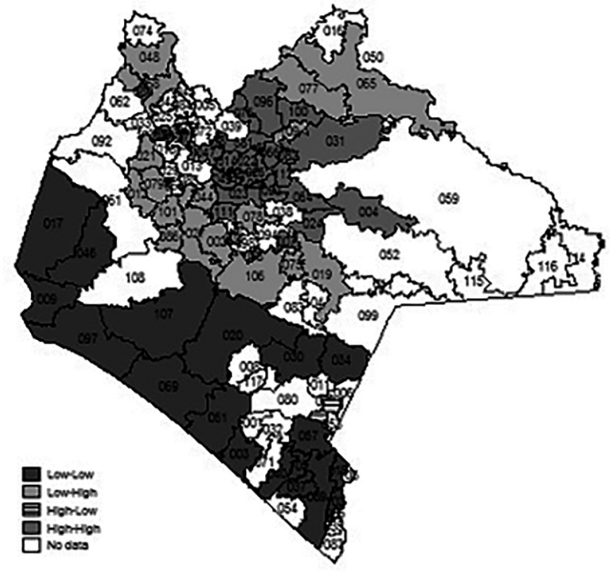

1.c. Fecundidad adolescente tardía $15-19$

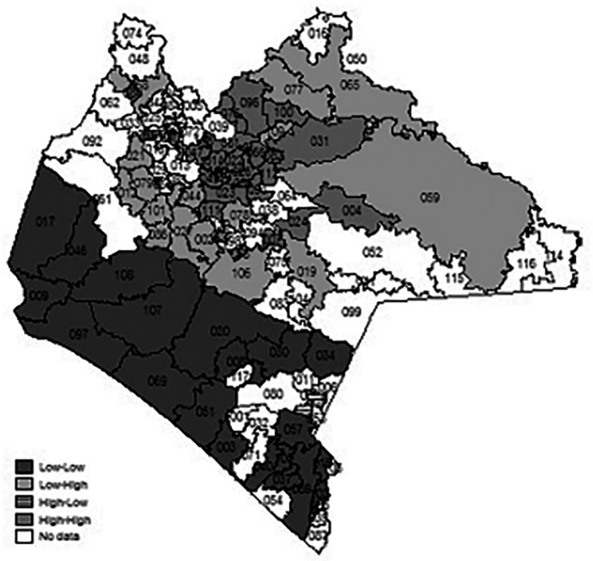

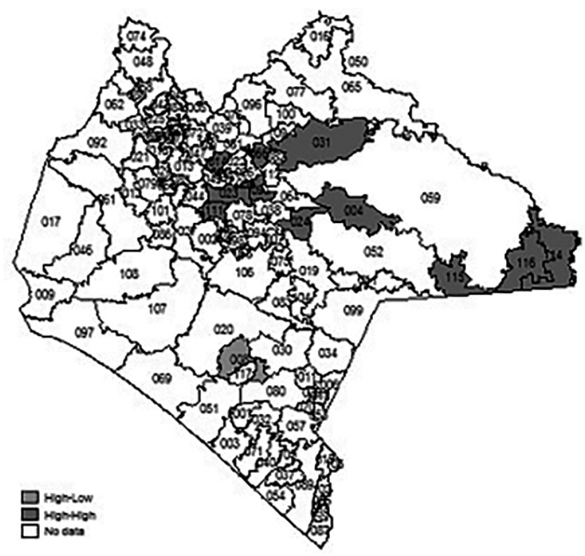

1.d. Fecundidad adolescente 10-19

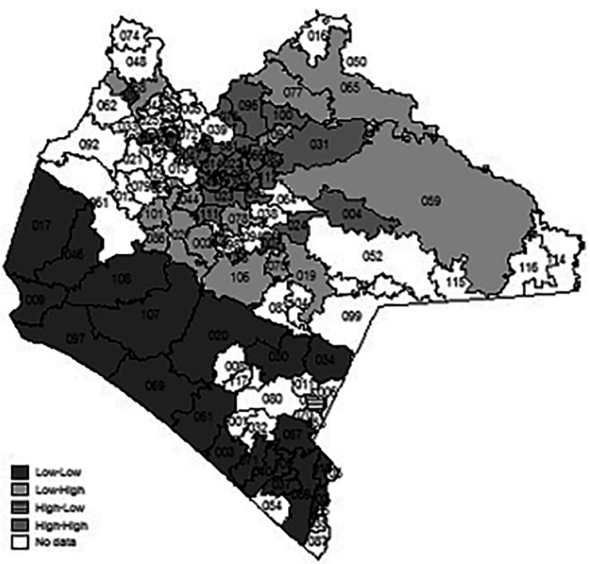

Fuente: elaboración propia con datos de la encuesta intercensal INEGI 2015, de CONAPO 2015. 
Mapas 2.a. a 2.d. Autocorrelación espacial de algunos determinantes de la fecundidad municipal en Chiapas

2.a. Índice de marginación

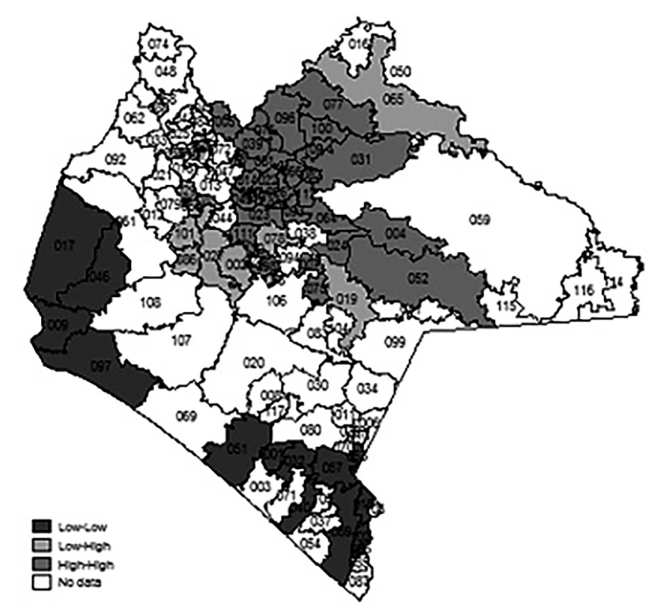

2.c. Escolaridad media

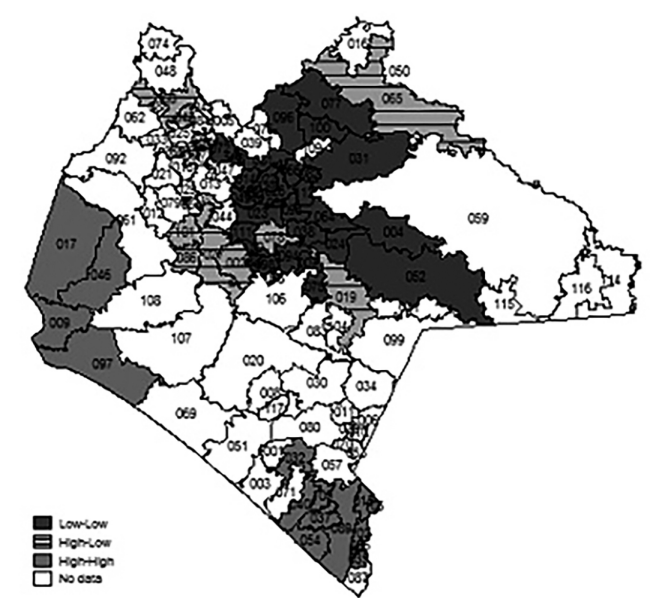

2.b. Población indígena femenina

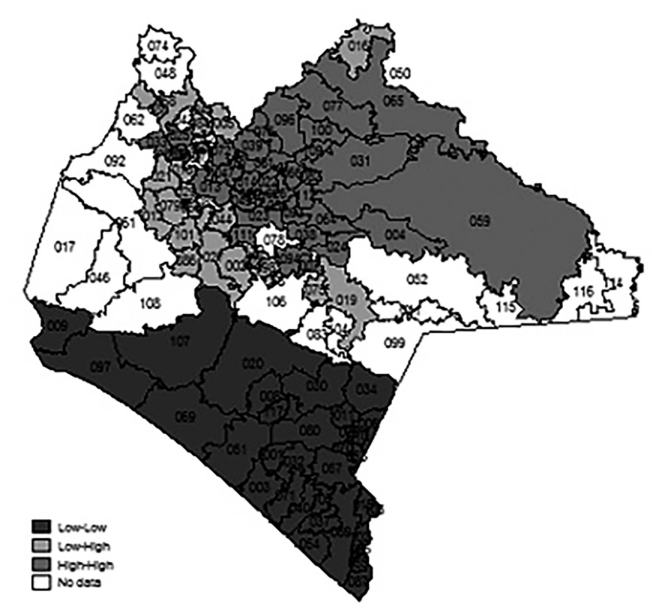

2.d. Analfabetismo femenino

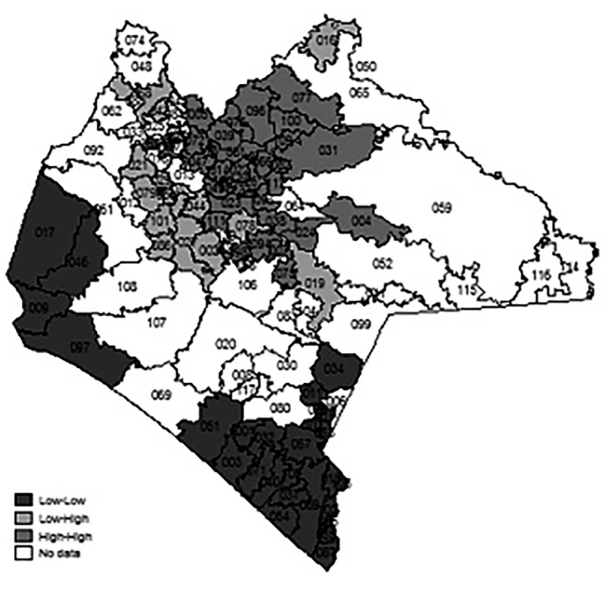

Fuente: elaboración propia con datos de la encuesta intercensal INEGI 2015, de CONAPO 2015. 